\title{
2D Octagon-Structure Carbon and Its Polarization Resolved Raman Spectra
}

\author{
Chunshan $\mathrm{He}^{1}\left[\right.$ and Weiliang Wang ${ }^{2, *}$ (]) \\ 1 State Key Laboratory of Optoelectronic Materials and Technologies, School of Physics, \\ Sun Yat-Sen University, Guangzhou 510275, China; stshcs@mail.sysu.edu.cn \\ 2 State Key Laboratory of Optoelectronic Materials and Technologies, School of Physics, Guangdong Province \\ Key Laboratory of Display Material and Technology, Sun Yat-Sen University, Guangzhou 510275, China \\ * Correspondence: wangwl2@mail.sysu.edu.cn
}

Received: 24 October 2020; Accepted: 11 November 2020; Published: 13 November 2020

check for updates

\begin{abstract}
We predict a new phase of two-dimensional carbon with density functional theory (DFT). It was found to be semimetal with two Dirac points. The vibrational properties and the polarization resolved Raman spectra of the carbon monolayer are predicted. There are five Raman active modes: $574 \mathrm{~cm}^{-1}\left(\mathrm{E}_{\mathrm{g}}\right), 1112 \mathrm{~cm}^{-1}\left(\mathrm{~B}_{1 \mathrm{~g}}\right), 1186 \mathrm{~cm}^{-1}\left(\mathrm{~B}_{2 \mathrm{~g}}\right), 1605 \mathrm{~cm}^{-1}\left(\mathrm{~B}_{2 \mathrm{~g}}\right)$ and $1734 \mathrm{~cm}^{-1}\left(\mathrm{~A}_{1 \mathrm{~g}}\right)$. We consider the incident light wave vector to be perpendicular and parallel to the plane of the carbon monolayer. By calculating Raman tensor of each Raman active mode, we obtained polarization angle dependent Raman intensities. Our results will help materials scientists to identify the existence and orientation of octagon-structure carbon monolayer when they are growing it.
\end{abstract}

Keywords: polarized Raman; vibrational properties; 2D carbon; DFT

\section{Introduction}

Graphene has become a hot scientific topic since 2004 [1]. A lot of theoretical and experimental results show that graphene has remarkable properties in mechanics, thermology, electronics and optics [2,3]. In recent years, people found many 2D materials from allotropes of carbon and from group V elements. Some of them have potential application in optoelectronic, nano electronic devices and hydrogen storage, because of their unique electronic and mechanical properties [4-10]. In 2018, Cao et al. found the twisted bilayer graphene is able to be an insulator or superconductor [11,12]. In the last few decades, scientists have sought and researched some other 2D materials for nanoelectronic, optoelectronic and thermoelectronic devices [13], such as hexagonal boron nitride ( $h$-BN) [14-16] and transition metal dichalcogenides (TMDCs) [17-22]. Zhang et al. predicted a novel 2D octagon-structure monolayer of nitrogen [23]. Its structure is composed of squares and octagons. There are many good electronic properties of this 2D material. It could be a potential candidate for use in the semiconductor devices, spintronics and quantum computation. Octagon-structure materials can be expected to be a good candidate for hydrogen storage. It is a possible high-temperature superconductor [24]. In 2015, Lu et al. fabricated free-standing octagon-shaped carbon nanofiber [25]. In 2017, Zhong's research group fabricated graphene-like nanoribbons which are periodically embedded with four- and eight-membered rings [26].

In this work, we theoretically predict a new phase of 2D carbon which consists of octagon carbon rings. Raman spectroscopy is a widely used experimental tool to characterize materials without destroying the samples $[27,28]$. People observed polarization dependent Raman intensity in many 2D materials, such as $\mathrm{WS}_{2}$ and $\mathrm{MoS}_{2}$ [29-32]. Therefore, we investigated the vibrational properties and predict the Raman spectra of this $2 \mathrm{D}$ octagon-structure carbon by density functional theory (DFT) to help materials scientists to identify the existence of this new phase of 2D carbon. 
In 2011, a carbon allotrope named as T-carbon was predicted by $\mathrm{Su}$ and his co-workers in theory [33]. Six year later, Zhang et al. synthesized T-carbon from pseudo-topotactic conversion of a multi-walled carbon nanotube suspension in methanol by picosecond pulsed-laser irradiation [34]. Therefore, the 2D Octagon-structure Carbon is holds much hope for synthesis in the future. This work sets a goal for materials scientists, providing a way for them to verify their experimental results, and providing a vast playground for theorists.

\section{Materials and Methods}

Geometry optimization, force constant calculation and dielectric tensor calculation were performed with the Vienna Ab initio simulation package (VASP, version 5.4.4., Wien, Austria) [35]. Phonon wavenumber and phonon modes were obtained with the Phonopy code [36]. The electron-core interactions were treated in the projector augmented wave (PAW) approximation [35,37-39]. The density functional was treated by generalized gradient approximation (GGA) with the Perdew-Burke-Ernzerhof (PBE) exchange correlation potential [40]. Kinetic energy cutoffs of $520 \mathrm{eV}$ and $400 \mathrm{eV}$ were used in geometry optimization and other calculations, respectively. The $k$ point mesh was $15 \times 15 \times 1$, $20 \times 20 \times 1$ and $51 \times 51 \times 1$ in geometry optimization, force constant calculation and dielectric function calculation, respectively. Vacuum slabs of $5 \mathrm{~nm}$ thick were inserted between neighboring $2 \mathrm{D}$ atom sheets. The convergence tolerance for the total energy and force calculations were set to $10^{-8} \mathrm{eV}$ and $10^{-5} \mathrm{eV} / \AA$, respectively.

The Raman tensor was obtained with the finite displacement method [31,32,41-52]:

$$
R_{\alpha \beta}(j)=V \sum_{\mu=1}^{N} \sum_{l=1}^{3} \frac{\partial \chi_{\alpha \beta}}{\partial r_{l}(\mu)} \frac{e_{l}^{j}(\mu)}{\sqrt{M_{\mu}}}
$$

where $V$ is the volume of unit cell, $M_{\mu}$ is the atomic mass of atom $\mu, \partial \chi_{\alpha \beta} / \partial r_{l}(\mu)$ is the first derivative of the electric polarizability tensor with respect to the atomic displacement. It equals the derivative of dielectric tensor $\varepsilon_{\alpha \beta}$ divided by $4 \pi$ because $\chi_{\alpha \beta}(\omega)=\left(\varepsilon_{\alpha \beta}(\omega)-\delta_{\alpha \beta}\right) / 4 \pi$. $\omega$ is the frequency of the laser (electric field). $e_{l}^{j}(\mu)$ is the eigenvector of the $\mathrm{j}$-th phonon mode at $\Gamma$ point. The dielectric tensor of the structure with finite displacement can be obtained with DFT.

Then Raman intensity can be obtained with [31,32]:

$$
\mathrm{I} \propto \omega_{s}^{4}\left|\vec{e}_{s} \cdot \stackrel{\leftrightarrow}{R} \cdot \vec{e}_{i}\right|^{2}
$$

where $\omega_{s}$ is the frequency of scattered light; $\vec{e}_{i}$ and $\vec{e}_{s}$ are electric polarization vectors of incident and scattered light, respectively.

\section{Results}

The unit cell of monolayer octagon-structure carbon consists of eight atoms (Figure 1a). The lattice constants: $a=b=4.87 \AA$; the bond length $l_{1}=1.373 \AA, l_{2}=1.466 \AA$. The buckled displacement $\Delta z$ is zero (Figure 1b). It belongs to space group P4/mmm (123). The stable lattice structures are shown in Figure 1. Figure 2 shows its band energy structures. It is a semimetal with two Dirac points.

Figure $3 \mathrm{a}$ is the phonon dispersion of the carbon monolayer. It does not have imaginary vibrating modes. The octagon-structure carbon monolayer belongs to the $\mathrm{D}_{4 \mathrm{~h}}$ point group, whose Raman active modes are $A_{1 g}$ (in-plane vibration), $B_{1 g}$ (in-plane vibration), $B_{2 g}$ (in-plane vibration) and $E_{g}$ (out-of-plane vibration). The atomic vibration displacements are shown in Figure $3 b$. The $\Gamma$ point wavenumbers of the $A_{1 \mathrm{~g}}$ mode and $B_{1 \mathrm{~g}}$ mode are $1734 \mathrm{~cm}^{-1}$ and $1112 \mathrm{~cm}^{-1}$, respectively. The $\Gamma$ point wavenumbers of the two $B_{2 g}$ modes are 1186 and $1605 \mathrm{~cm}^{-1}$. The $\Gamma$ point wavenumber of the $E_{g}$ mode is $574 \mathrm{~cm}^{-1}$. 


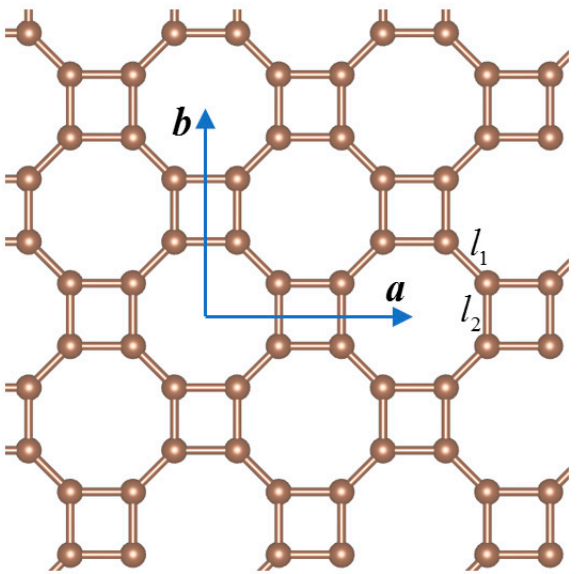

(a)

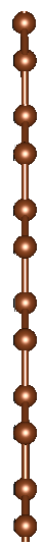

(b)

Figure 1. (a) Top view and (b) side view of the octagon-structure carbon monolayer. The blue arrows $\boldsymbol{a}$ and $\boldsymbol{b}$ show two basis vectors of the unit cell which are along the $x$-axis and $y$-axis, respectively. The bond lengths $l_{1}$ and $l_{2}$ are the short and long edges of the octagons.

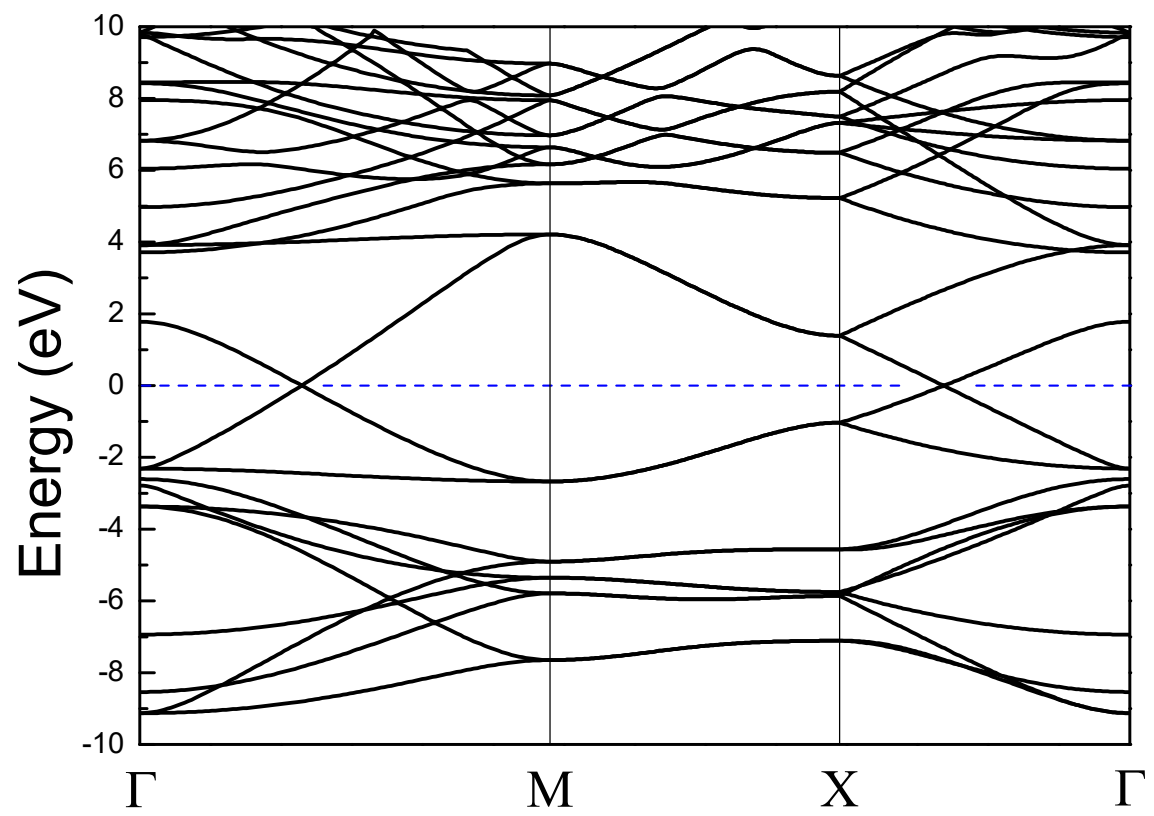

Figure 2. Band structure of the octagon-structure carbon monolayer.

In order to calculate the Raman intensity, we chose three commonly used laser lines whose wavelengths are $488 \mathrm{~nm}(2.54 \mathrm{eV}), 532 \mathrm{~nm}(2.33 \mathrm{eV})$ and $633 \mathrm{~nm}(1.96 \mathrm{eV})$ [27,30]. The coordinate system is chosen so that the monolayer plane is in the $x-y$ plane. The calculated results show that the Raman tensors agree with the point group $\mathrm{D}_{4 \mathrm{~h}}$ and their irreducible representations. They are the following matrix forms: 


$$
\begin{aligned}
& \widetilde{R}\left(A_{1 g}\right)=\left(\begin{array}{ccc}
a & 0 & 0 \\
0 & a & 0 \\
0 & 0 & b
\end{array}\right) \\
& \widetilde{R}\left(B_{1 g}\right)=\left(\begin{array}{ccc}
c & 0 & 0 \\
0 & -c & 0 \\
0 & 0 & 0
\end{array}\right) \\
& \widetilde{R}\left(B_{2 g}\right)=\left(\begin{array}{lll}
0 & d & 0 \\
d & 0 & 0 \\
0 & 0 & 0
\end{array}\right) \\
& \widetilde{R}\left(E_{g}\right)=\left(\begin{array}{lll}
0 & 0 & e \\
0 & 0 & f \\
e & f & 0
\end{array}\right)
\end{aligned}
$$

where $a, b, c, d, e, f$ are complex numbers.

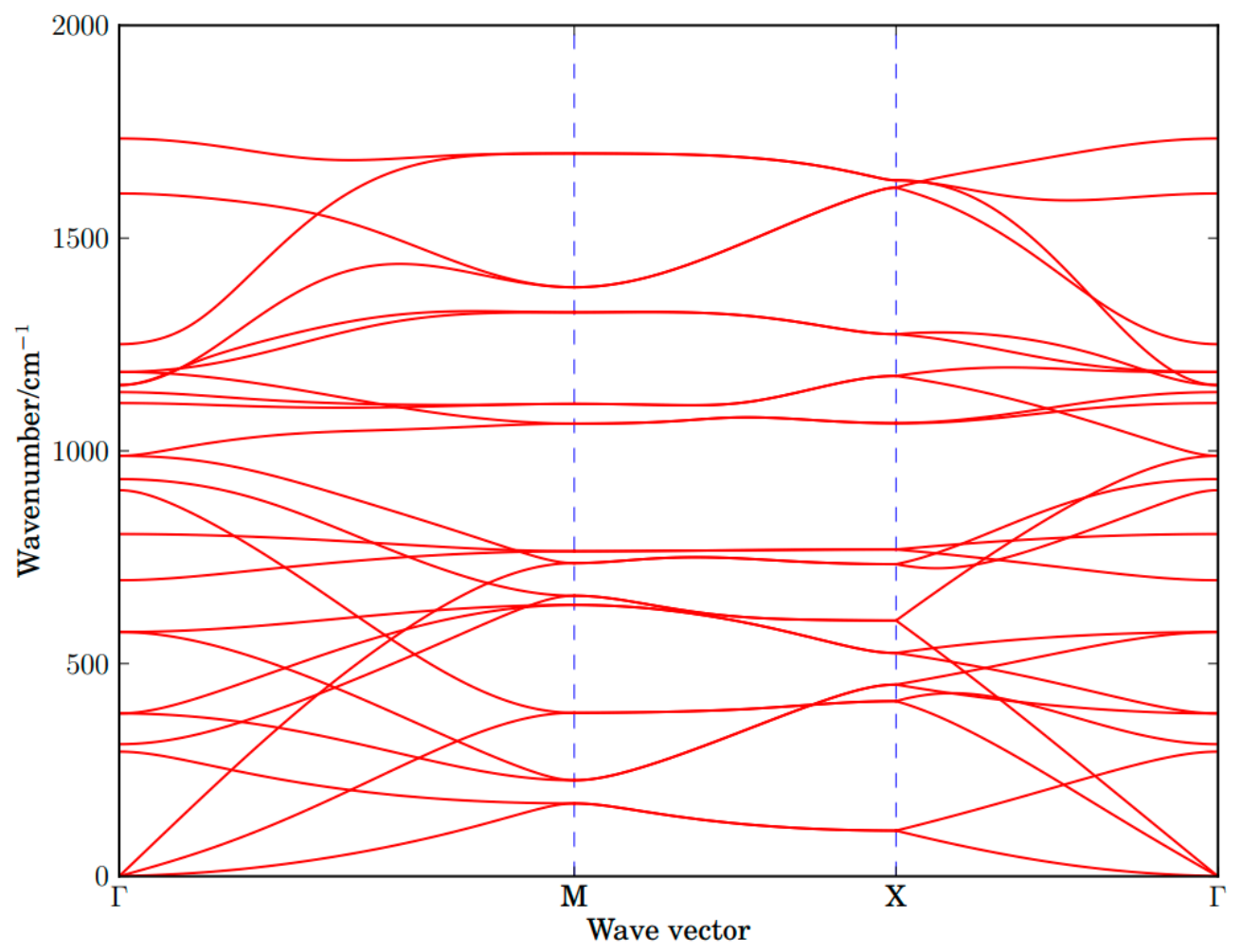

(a)
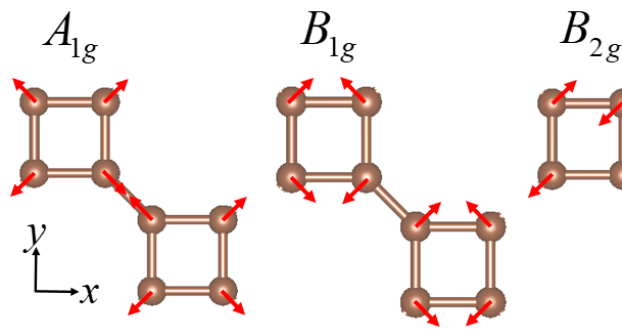

$B_{2 g}$

$E_{g}$
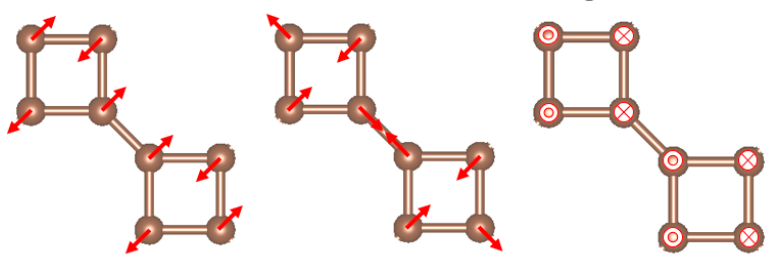

(b)

Figure 3. (a) Phonon dispersions of the octagon-structure carbon monolayer, (b) Raman active modes $A_{1 g}$ (in-plane), $B_{1 g}$ (in-plane), $B_{2 g}$ (in-plane) and $E_{g}$ (out-of-plane) at $\Gamma$ point. The dot and cross on the atoms refer to displacement in and out of the plane, respectively, and the red arrows refer to the in-plane displacement. The length of the arrows is nonphysical. 
When the incident light wave vector is perpendicular to the monolayer plane ( $x-y$ plane), the polarization direction of the incident light is in the $x-y$ plane and can be written as $e_{i}=(\cos \theta, \sin \theta, 0)$. We considered two polarization directions of scattered light which are parallel and perpendicular to the polarization direction of the incident light, called parallel polarization configuration and perpendicular polarization configuration, respectively. Then the polarization directions of the scattered light can be written as $e_{s}^{\|}=(\cos \theta, \sin \theta, 0)$ and $e_{s}^{\perp}=(-\sin \theta, \cos \theta, 0)$, respectively. Based on the Raman tensors matrix and polarization directions of the incident and scattered light, we can obtain the polarization angle dependent Raman intensity for each mode. The Raman intensities for these polarization configurations are:

$$
\begin{gathered}
I^{\|}\left(A_{1 g}\right) \propto|a|^{2} \\
I^{\|}\left(B_{1 g}\right) \propto|c|^{2} \cos ^{2}(2 \theta) \\
I^{\|}\left(B_{2 g}\right) \propto|d|^{2} \sin ^{2}(2 \theta) \\
I^{\|}\left(E_{g}\right) \propto 0 \\
I^{\perp}\left(A_{1 g}\right) \propto 0 \\
I^{\perp}\left(B_{1 g}\right) \propto|c|^{2} \sin ^{2}(2 \theta) \\
I^{\perp}\left(B_{2 g}\right) \propto|d|^{2} \cos ^{2}(2 \theta) \\
I^{\perp}\left(E_{g}\right) \propto 0
\end{gathered}
$$

For the $\mathrm{A}_{1 \mathrm{~g}}$ mode, Equations (4) and (8) mean that the scattered lights intensity is independent of the polarization angle $\theta$. The intensity is zero for the $\mathrm{E}_{\mathrm{g}}$ mode. Only the $\mathrm{B}_{1 \mathrm{~g}}$ modes and $\mathrm{B}_{2 \mathrm{~g}}$ modes show the polarization angle dependent intensity. The polarization dependent Raman intensities of the $B_{1 \mathrm{~g}}$ modes and $\mathrm{B}_{2 \mathrm{~g}}$ modes of the carbon monolayer for three laser lines $488 \mathrm{~nm}, 532 \mathrm{~nm}$ and $633 \mathrm{~nm}$, when the wave vector of the laser is perpendicular to the 2D plane, are shown in Figure 4 (parallel polarization configuration) and Figure 5 (perpendicular polarization configuration). The order of magnitude of Raman intensities excited by different laser lines is different because the Raman intensity is proportional to $\omega_{s}^{4}$ (Equation (2)). Therefore, we plot $\left|\vec{e}_{S} \cdot \stackrel{\leftrightarrow}{R} \cdot \vec{e}_{i}\right|^{2}$ for clarity.

From Figure 4 , Raman intensities of the $B_{1 g}$ and $B_{2 g}$ modes in parallel polarization configurations have four maxima and four minima for all the laser lines. Due to the factor $\cos ^{2}(2 \theta)$ in Equation (5), the maxima of Raman intensities of $\mathrm{B}_{1 \mathrm{~g}}$ modes (Figure $\left.4 \mathrm{a}\right)$ locate at $\theta=0^{\circ}+n \times 90^{\circ}(n=0,1,2,3)$; the four minima locate at $\theta=45^{\circ}+n \times 90^{\circ}(n=0,1,2,3)$. Due to the factor $\sin ^{2}(2 \theta)$ in Equation (6), the maxima of Raman intensities of $\mathrm{B}_{2 \mathrm{~g}}$ modes (Figure $\left.4 \mathrm{~b}, \mathrm{c}\right)$ locate at $\theta=45^{\circ}+n \times 90^{\circ}(n=0,1,2,3)$; the four minima locate at $\theta=0^{\circ}+n \times 90^{\circ}(n=0,1,2,3)$. The location of the maxima and minima in Figure 5 are result from a similar reason to that in Figure 4 . The polarization angle dependent Raman intensity with the incident light wave vector in the $\mathrm{z}$ direction is different from some other isotropic 2D materials (e.g., $\beta$-Arsenic monolayer [47] and $M X$ enes $Z_{2} X(X=C$ and $N)$ [49]). The anisotropic vibrations are responsible for the polarization dependent Raman intensity. 

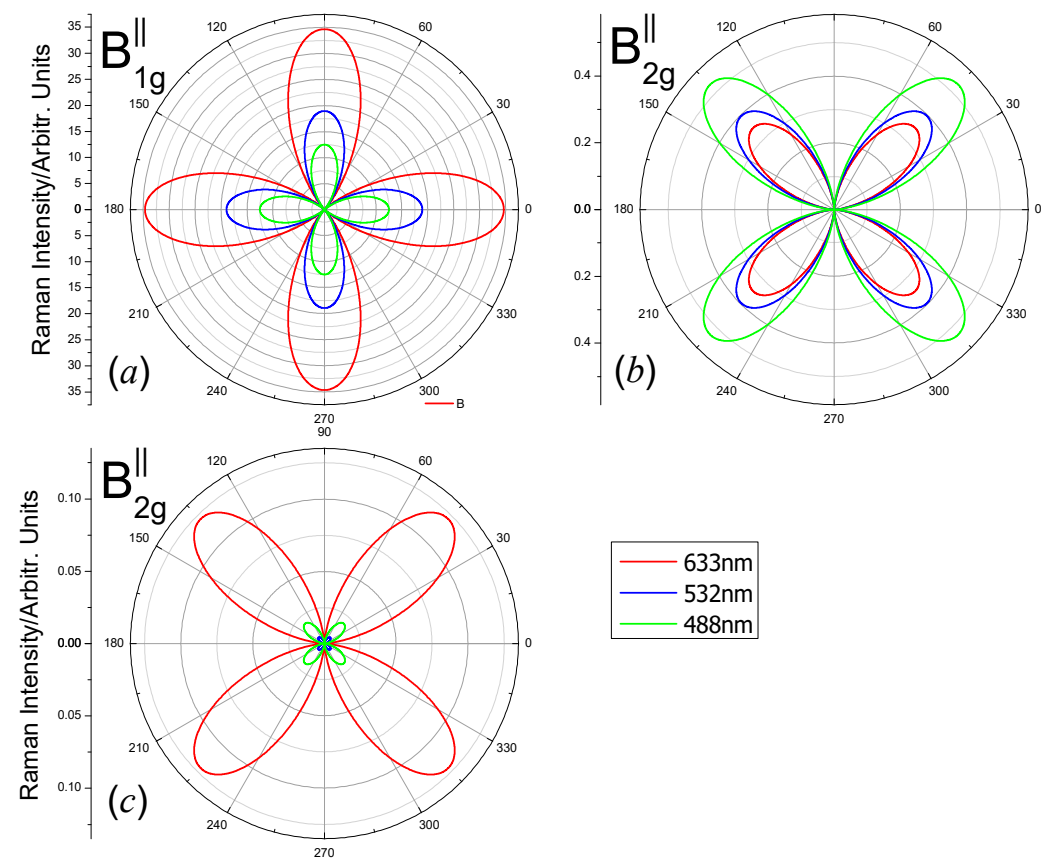

Figure 4. Polar plots of the angular dependent Raman intensities $\left|\overrightarrow{e_{S}} \cdot \stackrel{\leftrightarrow}{R} \cdot \vec{e}_{i}\right|^{2}$ of the (a) $\mathrm{B}_{1 \mathrm{~g}}-1112 \mathrm{~cm}^{-1}$, (b) $\mathrm{B}_{2 \mathrm{~g}}-1605 \mathrm{~cm}^{-1}$ and (c) $\mathrm{B}_{2 \mathrm{~g}}-1186 \mathrm{~cm}^{-1}$ modes of the octagon-structure carbon monolayer excited by laser lines $488 \mathrm{~nm}$ (green), $532 \mathrm{~nm}$ (blue) and $633 \mathrm{~nm}$ (red). The polarization direction of the scattered light is parallel to the polarization direction of the incident light. The incident and scattered light wave vectors are in the $z$-direction.
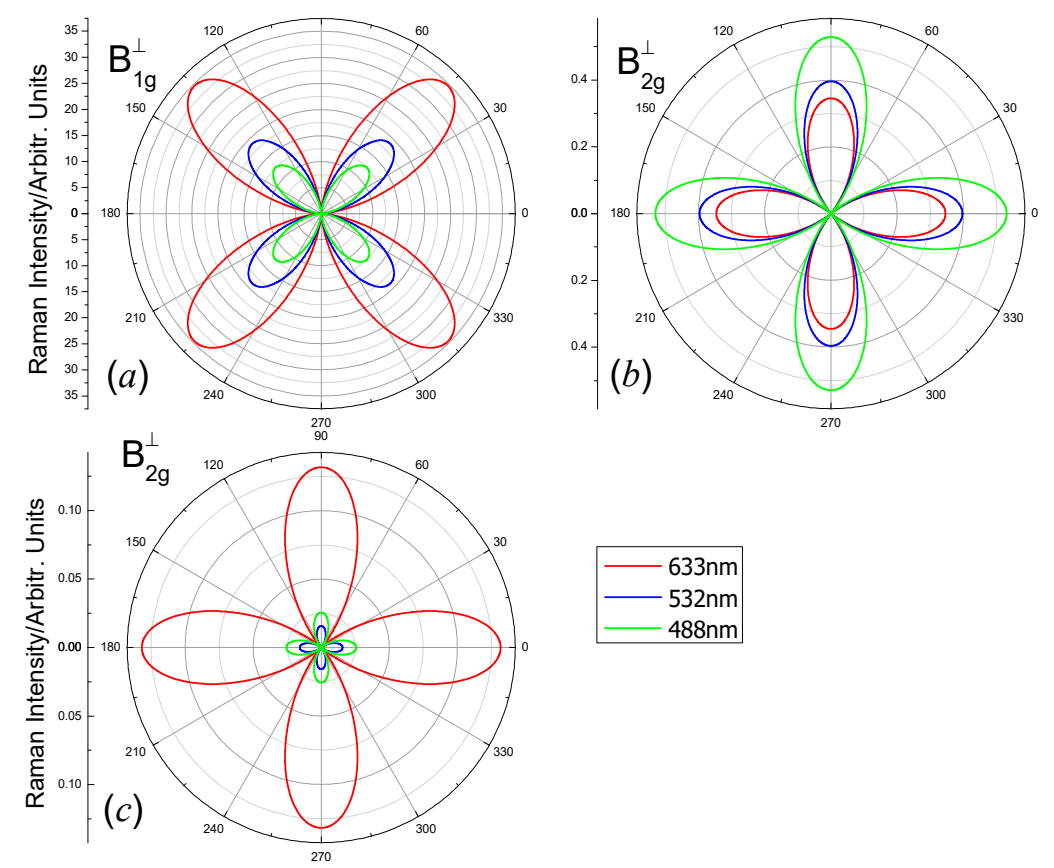

Figure 5. Polar plots of the angular dependent Raman intensities $\left|\vec{e}_{s} \cdot \stackrel{\leftrightarrow}{R} \cdot \vec{e}_{i}\right|^{2}$ of (a) $\mathrm{B}_{1 \mathrm{~g}}-1112 \mathrm{~cm}^{-1}$, (b) $\mathrm{B}_{2 \mathrm{~g}}-1605 \mathrm{~cm}^{-1}$ and (c) $\mathrm{B}_{2 \mathrm{~g}}-1186 \mathrm{~cm}^{-1}$ modes of the octagon-structure carbon monolayer excited by laser lines $488 \mathrm{~nm}$ (green), $532 \mathrm{~nm}$ (blue) and $633 \mathrm{~nm}$ (red). The polarization direction of the scattered light is perpendicular to the polarization direction of the incident light. The incident and scattered light wave vectors are in the $z$-direction. 
If the incident light wave vector is along the $x$-axis, the polarization direction of the incident light is in the $y-z$ plane and can be written as $e_{i}=(0, \cos \theta, \sin \theta)$. We also considered parallel polarization configuration and perpendicular polarization configuration, in which the polarization directions of the scattered light can be written as $e_{s}^{\|\|}=(0, \cos \theta, \sin \theta)$ and $e_{s}^{\perp}=(0,-\sin \theta, \cos \theta)$, respectively. Based on the Raman tensors matrix and polarization directions of the incident light and scattered light, we can obtain the polarization angle dependent Raman intensity for each mode. The Raman intensities are:

$$
\begin{gathered}
I^{\|}\left(A_{1 g}\right) \propto|a|^{2} \cos ^{4} \theta+|b|^{2} \sin ^{4} \theta+\operatorname{Re}\left(a \cdot b^{*}\right) \sin ^{2}(2 \theta) / 2 \\
I^{\|}\left(B_{1 g}\right) \propto|c|^{2} \cos ^{4} \theta \\
I^{\|}\left(B_{2 g}\right) \propto 0 \\
I^{\|}\left(E_{g}\right) \propto|f|^{2} \sin ^{2}(2 \theta) \\
I^{\perp}\left(A_{1 g}\right) \propto\left[|a|^{2}+|b|^{2}-2 \operatorname{Re}\left(a \cdot b^{*}\right)\right] \sin ^{2}(2 \theta) / 4 \\
I^{\perp}\left(B_{1 g}\right) \propto|c|^{2} \sin ^{2}(2 \theta) / 4 \\
I^{\perp}\left(B_{2 g}\right) \propto 0 \\
I^{\perp}\left(E_{g}\right) \propto|f|^{2} \cos ^{2}(2 \theta)
\end{gathered}
$$

The angular dependent Raman intensities of different vibrating modes of the carbon monolayer for three laser lines $488 \mathrm{~nm}, 532 \mathrm{~nm}$ and $633 \mathrm{~nm}$, when the wave vector is parallel to the 2D plane, are shown in Figure 6 (parallel polarization configuration) and Figure 7 (perpendicular polarization configuration).
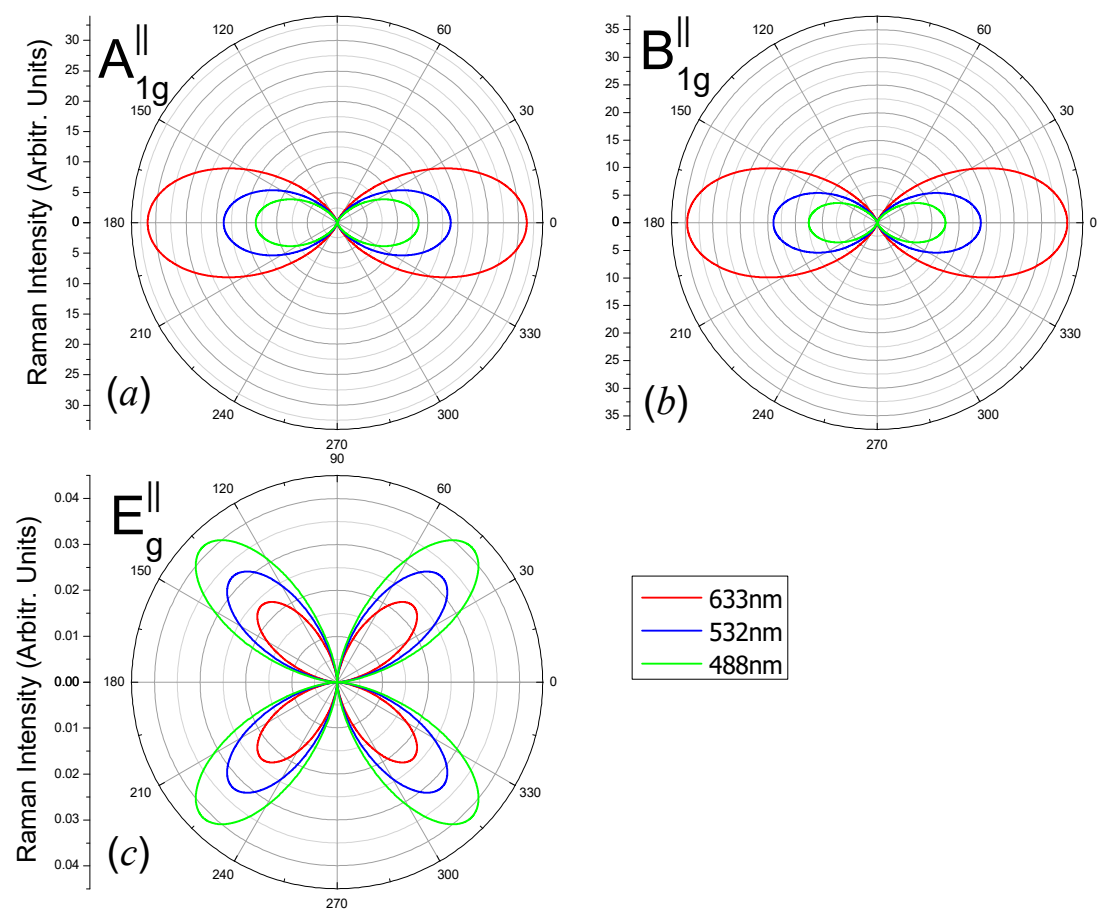

Figure 6. Polar plots of the angular dependent Raman intensities $\left|\vec{e}_{s} \cdot \stackrel{\leftrightarrow}{R} \cdot \vec{e}_{i}\right|^{2}$ of (a) $\mathrm{A}_{1 \mathrm{~g}}-1734 \mathrm{~cm}^{-1}$, (b) $\mathrm{B}_{1 \mathrm{~g}}-1112 \mathrm{~cm}^{-1}$ and (c) $\mathrm{E}_{\mathrm{g}}-574 \mathrm{~cm}^{-1}$ modes of the octagon-structure carbon monolayer excited by laser lines $488 \mathrm{~nm}$ (green), $532 \mathrm{~nm}$ (blue) and $633 \mathrm{~nm}$ (red). The polarization direction of the scattered light is parallel to the polarization direction of the incident light. The incident and scattered light wave vectors are in the $x$-direction. 

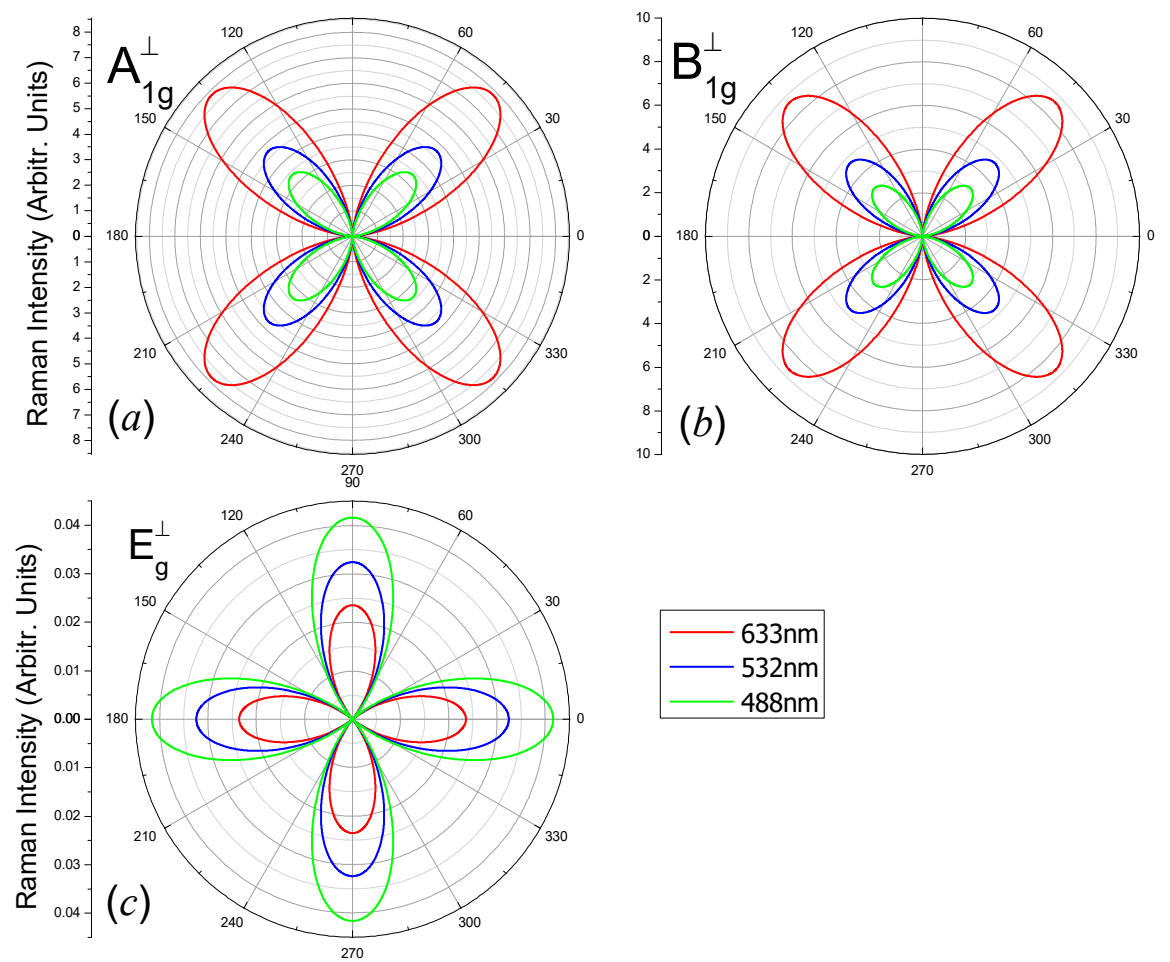

Figure 7. Polar plots of the angular dependent Raman intensities $\left|\vec{e}_{s} \cdot \stackrel{\leftrightarrow}{R} \cdot \vec{e}_{i}\right|^{2}$ of (a) $\mathrm{A}_{1 \mathrm{~g}}-1734 \mathrm{~cm}^{-1}$, (b) $B_{1 \mathrm{~g}}-1112 \mathrm{~cm}^{-1}$ and (c) $\mathrm{E}_{\mathrm{g}}-574 \mathrm{~cm}^{-1}$ modes of the octagon-structure carbon monolayer excited by laser lines $488 \mathrm{~nm}$ (green), $532 \mathrm{~nm}$ (blue) and $633 \mathrm{~nm}$ (red). The polarization direction of the scattered light is perpendicular to the polarization direction of the incident light. The incident and scattered light wave vectors are in the $x$-direction.

From Figure 6, the maxima locate at $0^{\circ}$ and $180^{\circ}$ for the $A_{1 g}$ modes (Figure 6a), because $|a|$ is always much larger than $|b|$, which may change with laser photon energy; the maxima locate at $0^{\circ}$ and $180^{\circ}$ for $\mathrm{B}_{1 \mathrm{~g}}$ modes (Figure $6 \mathrm{~b}$ ) due to the factor $\cos ^{4} \theta$; the Raman intensities have four maxima which locate at $\theta=45^{\circ}+n \times 90^{\circ}(n=0,1,2,3)$ for the $E_{g}$ mode (Figure 6c) due to the factor $\sin ^{2}(2 \theta)$.

From Figure $7 \mathrm{a}, \mathrm{b}$, Raman intensities of the $\mathrm{A}_{1 \mathrm{~g}}$ and $\mathrm{B}_{1 \mathrm{~g}}$ modes in perpendicular polarization configurations have four maxima for all the laser lines; the maxima intensities locate at $\theta=45^{\circ}+n \times$ $90^{\circ}(n=0,1,2,3)$ and the minima ones are zero at $\theta=0^{\circ}+n \times 90^{\circ}(n=0,1,2,3)$, due to the factor $\sin ^{2}(2 \theta)$. The Raman intensities have four maxima which locate at $\theta=0^{\circ}+n \times 90^{\circ}(n=0,1,2,3)$ for the $\mathrm{E}_{\mathrm{g}}$ mode (Figure $7 \mathrm{c}$ ) due to the factor $\cos ^{2}(2 \theta)$.

By integrating the Raman intensity of all polarization directions, we obtained the monolayer's nonpolarized Raman intensity of the monolayer when the incident light wave vector is the along $z$ and $x$ direction (Figure 8). The factor $\omega_{s}^{4}$ in Equation (2) is omitted in Figure 8 for clarity.

When the incident light is along the $z$-axis, the monolayer has four distinct peaks (Figure 8a) at $1112 \mathrm{~cm}^{-1}\left(\mathrm{~B}_{1 \mathrm{~g}}\right), 1186 \mathrm{~cm}^{-1}\left(\mathrm{~B}_{2 \mathrm{~g}}\right), 1605 \mathrm{~cm}^{-1}\left(\mathrm{~B}_{2 \mathrm{~g}}\right)$ and $1734 \mathrm{~cm}^{-1}\left(\mathrm{~A}_{1 \mathrm{~g}}\right)$. When the incident light is along the $x$-axis, the monolayer has three peaks (Figure $8 \mathrm{~b}$ ) at $574 \mathrm{~cm}^{-1}\left(\mathrm{E}_{\mathrm{g}}\right), 1112 \mathrm{~cm}^{-1}\left(\mathrm{~B}_{1 \mathrm{~g}}\right)$ and $1734 \mathrm{~cm}^{-1}\left(\mathrm{~A}_{1 \mathrm{~g}}\right)$. Whatever the direction of the incident light, the Raman intensity of $1112 \mathrm{~cm}^{-1}\left(\mathrm{~B}_{1 \mathrm{~g}}\right)$ and $1734 \mathrm{~cm}^{-1}\left(\mathrm{~A}_{1 \mathrm{~g}}\right)$ are always the strongest and the second strongest.

We calculated the energy of the octagon 2D carbon and compare it with the other allotrope: graphene, graphite and diamond (Table 1). It is found that the octagon carbon monolayer's energy is greater than graphene and diamond. It means graphene, graphite and diamond are more stable than the octagon 2D carbon. 


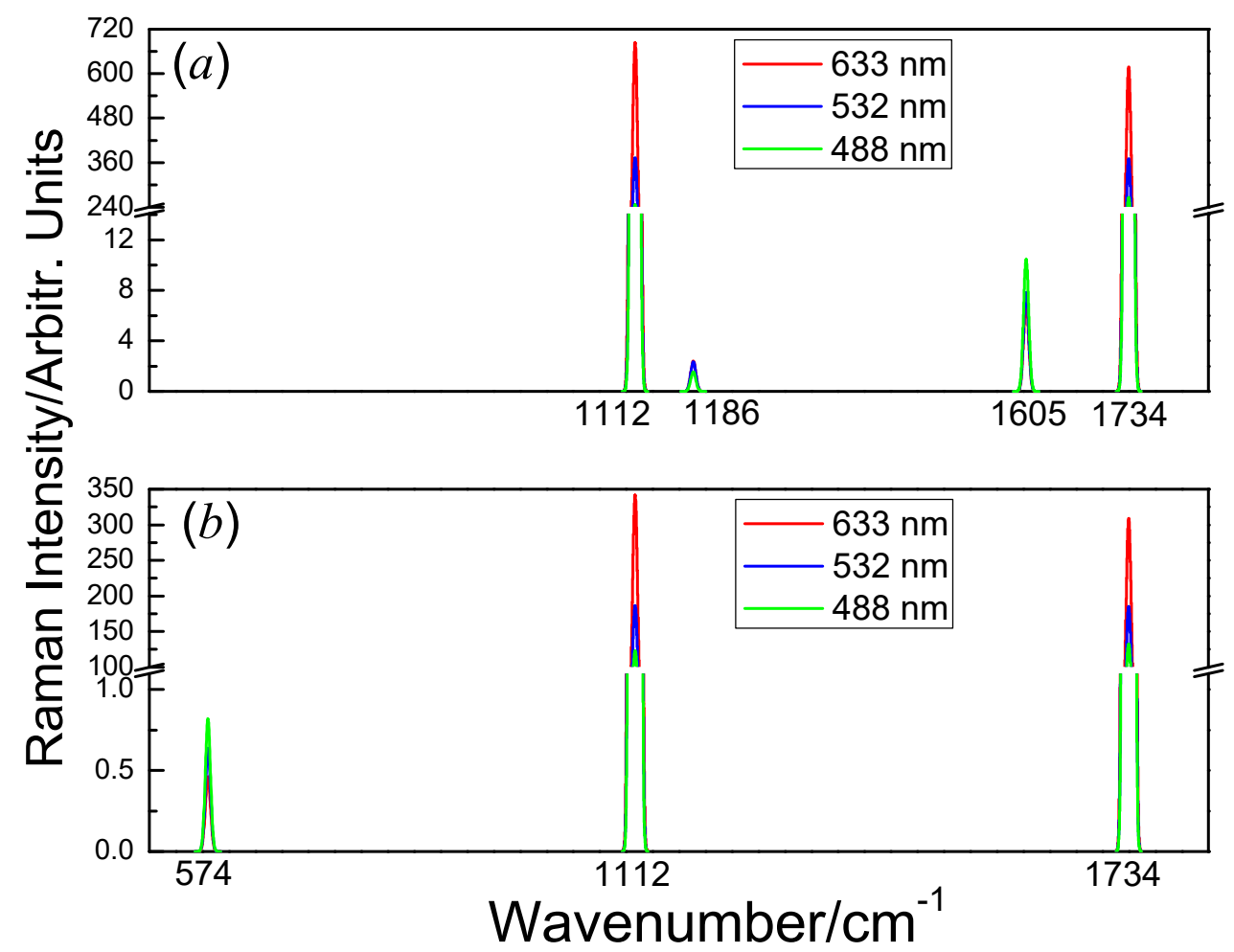

Figure 8. Raman spectra of the octagon-structure carbon monolayer for three laser lines: $488 \mathrm{~nm}$ (green), $532 \mathrm{~nm}$ (blue) and $633 \mathrm{~nm}$ (red) with a Gaussian broadening width of $3 \mathrm{~cm}^{-1}$. (a) The incident light is from $z$-axis. (b) The incident light is from $x$-axis.

Table 1. Free energy of graphene, graphite, diamond and octagon carbon.

\begin{tabular}{cc}
\hline Material & Average Energy/Atom \\
\hline Graphene & $-9.224 \mathrm{eV}$ \\
\hline Octagon Carbon monolayer & $-8.711 \mathrm{eV}$ \\
\hline Graphite & $-9.306 \mathrm{eV}$ \\
\hline Diamond & $-9.099 \mathrm{eV}$ \\
\hline Octagon Carbon Bulk & $-8.782 \mathrm{eV}$ \\
\hline
\end{tabular}

\section{Discussion and Conclusions}

The Raman shifts for graphene and graphite are 1350, 1600 and $2700 \mathrm{~cm}^{-1}$ [53,54]; the Raman shifts for diamond are 1332, 2015 and $2176 \mathrm{~cm}^{-1}$ [55]. The strong and distinct $1112 \mathrm{~cm}^{-1}\left(\mathrm{~B}_{1 \mathrm{~g}}\right)$ and $1734 \mathrm{~cm}^{-1}\left(\mathrm{~A}_{1 \mathrm{~g}}\right)$ Raman shifts of octogen 2D carbon are unique. They can help materials scientists to distinguish it from graphene, graphite and diamond.

There are five Raman active modes: $574 \mathrm{~cm}^{-1}\left(\mathrm{E}_{\mathrm{g}}\right), 1112 \mathrm{~cm}^{-1}\left(\mathrm{~B}_{1 \mathrm{~g}}\right), 1186 \mathrm{~cm}^{-1}\left(\mathrm{~B}_{2 \mathrm{~g}}\right), 1605 \mathrm{~cm}^{-1}\left(\mathrm{~B}_{2 \mathrm{~g}}\right)$ and $1734 \mathrm{~cm}^{-1}\left(\mathrm{~A}_{1 \mathrm{~g}}\right)$. When the incident light wave vector is perpendicular to the plane of the carbon monolayer, $\mathrm{B}_{1 \mathrm{~g}}$ and $\mathrm{B}_{2 \mathrm{~g}}$ modes can show polarization-angle-dependent Raman intensity. This is different from some other isotropic 2D materials (e.g., $\beta$-Arsenic monolayer [47], $\mathrm{Zr}_{2} \mathrm{C}$ monolayer and $\mathrm{Zr}_{2} \mathrm{~N}$ monolayer [49]). There all are four maxima and minima for all the laser lines when the polarization direction of the scattered light is parallel or perpendicular to that of the incident light. The incident light wave vector is parallel to the plane of the carbon monolayer. For the $A_{1 g}$ and $B_{1 g}$ modes, Raman intensities have two (four) minima and two (four) maxima when the polarization direction of scattered light is parallel (perpendicular) to that of the incident light. The intensity is 
zero for the $B_{2 g}$ mode. For the $E_{g}$ mode, there always are four minima and four maxima when the polarization direction of the scattered light is parallel or perpendicular to that of the incident light.

The Dirac cone may result in many excellent physical properties, such as very high carrier mobility and an anomalous quantum Hall effect. Due to the two-phonon double resonance Raman process between Dirac points, there will be some double resonant bands whose Raman shifts are approximately twice the $\Gamma$ point phonon frequencies $[54,56]$.

In conclusion, we predicted a new phase of 2D carbon: 2D octagon-structure carbon. It is found to be semimetal with two Dirac points. We investigated its vibrational properties and polarization resolved Raman spectra excited by three commonly used laser lines $488 \mathrm{~nm}, 532 \mathrm{~nm}$ and $633 \mathrm{~nm}$. The Raman spectra has four and three distinct peaks when the incident light wave vector is perpendicular and parallel to the carbon monolayer, respectively. We expect this octagon-structure carbon can be grown in experiments and would have wide applications.

Author Contributions: Conceptualization, C.H. and W.W.; methodology, C.H. and W.W.; software, C.H.; validation, W.W.; formal analysis, C.H. and W.W.; investigation, C.H. and W.W.; data curation, C.H.; writing-original draft preparation, C.H.; writing - review and editing, W.W. All authors have read and agreed to the published version of the manuscript.

Funding: This work was supported by Key-Area Research and Development Program of GuangDong Province (2019B030330001), National Natural Science Foundation of China (Grant No. 11704419), National R\&D Key Plan Project of China (2016YFA0202000), Guangzhou Science Technology and Innovation Commission (grant no. 201607020012), Natural Science Foundation of Guangdong Province (2018B030311045) and Physical Research Platform (PRP) in School of Physics, SYSU.

Conflicts of Interest: The authors declare no conflict of interest.

\section{References}

1. Novoselov, K.S.; Geim, A.K.; Morozov, S.V.; Jiang, D.; Zhang, Y.; Dubonos, S.V.; Grigorieva, I.V.; Firsov, A.A. Electric Field Effect in Atomically Thin Carbon Films. Science 2004, 306, 666-669. [CrossRef] [PubMed]

2. $\mathrm{Mu}, \mathrm{X}$.; Sun, M. The linear and non-linear optical absorption and asymmetrical electromagnetic interaction in chiral twisted bilayer graphene with hybrid edges. Mater. Today Phys. 2020, 14, 100222. [CrossRef]

3. Wang, J.; Mu, X.; Wang, L.; Sun, M. Properties and applications of new superlattice: Twisted bilayer graphene. Mater. Today Phys. 2019, 9, 100099. [CrossRef]

4. Li, P.; Luo, W. A new structure of two-dimensional allotropes of group V elements. Sci. Rep. 2016, 6, 25423. [CrossRef] [PubMed]

5. Sharma, B.R.; Manjanath, A.; Singh, A.K. pentahexoctite: A new two-dimensional allotrope of carbon. Sci. Rep. 2014, 4, 7164. [CrossRef] [PubMed]

6. Lin, W.; Liang, S.-D.; He, C.; Xie, W.; He, H.; Mai, Q.; Li, J.; Yao, D.-X. Stabilities and novel electronic structures of three carbon nitride bilayers. Sci. Rep. 2019, 9, 1-9. [CrossRef] [PubMed]

7. Bhattacharya, D.; Jana, D. First-principles calculation of the electronic and optical properties of a new two-dimensional carbon allotrope: Tetra-penta-octagonal graphene. Phys. Chem. Chem. Phys. 2019, 21, 24758-24767. [CrossRef]

8. Du, Q.-S.; Tang, P.-D.; Huang, H.-L.; Du, F.-L.; Huang, K.; Xie, N.-Z.; Long, S.-Y.; Li, Y.-M.; Qiu, J.-S.; Huang, R.-B. A new type of two-dimensional carbon crystal prepared from 1,3,5-trihydroxybenzene. Sci. Rep. 2017, 7, 40796. [CrossRef]

9. Lin, W.; Li, J.; Wang, W.; Liang, S.-D.; Yao, D.-X. Electronic Structure and Band Gap Engineering of Two-Dimensional Octagon-Nitrogene. Sci. Rep. 2018, 8, 1-8. [CrossRef]

10. Qin, G.; Hao, K.-R.; Yan, Q.-B.; Hu, M.; Su, G. Exploring T-carbon for energy applications. Nanoscale 2019, 11, 5798-5806. [CrossRef]

11. Cao, Y.; Fatemi, V.; Demir, A.; Fang, S.; Tomarken, S.L.; Luo, J.Y.; Sanchez-Yamagishi, J.D.; Watanabe, K.; Taniguchi, T.; Kaxiras, E.; et al. Correlated insulator behaviour at half-filling in magic-angle graphene superlattices. Nat. Cell Biol. 2018, 556, 80-84. [CrossRef] [PubMed]

12. Cao, Y.; Fatemi, V.; Fang, S.; Watanabe, K.; Taniguchi, T.; Kaxiras, E.; Jarillo-Herrero, P. Unconventional superconductivity in magic-angle graphene superlattices. Nat. Cell Biol. 2018, 556, 43-50. [CrossRef] [PubMed] 
13. Li, L.; Yu, Y.; Ye, G.J.; Ge, Q.; Ou, X.; Wu, H.; Feng, D.; Chen, X.H.; Zhang, Y. Black phosphorus field-effect transistors. Nat. Nanotechnol. 2014, 9, 372-377. [CrossRef] [PubMed]

14. Bhattacharya, A.; Das, G.P. Band gap engineering by functionalization of BN sheet. Phys. Rev. B 2012, 85, 035415. [CrossRef]

15. Slotman, G.J.; De Wijs, G.A.; Fasolino, A.; Katsnelson, M.I. Phonons and electron-phonon coupling in graphene-h-BN heterostructures. Ann. Phys. 2014, 526, 381-386. [CrossRef]

16. Wang, J.; Ma, F.; Liang, W.; Sun, M. Electrical properties and applications of graphene, hexagonal boron nitride (h-BN), and graphene/h-BN heterostructures. Mater. Today Phys. 2017, 2, 6-34. [CrossRef]

17. Deng, Z.; Li, Z.; Wang, W. Electron affinity and ionization potential of two-dimensional honeycomb sheets: A first principle study. Chem. Phys. Lett. 2015, 637, 26-31. [CrossRef]

18. Luo, Y.; Wang, S.; Tang, W.; Chou, J.-P.; Yu, J.; Sun, Z.; Sun, M. Transition-metal dichalcogenides/Mg $(\mathrm{OH})_{2}$ van der Waals heterostructures as promising water-splitting photocatalysts: A first-principles study. Phys. Chem. Chem. Phys. 2019, 21, 1791-1796. [CrossRef]

19. Yin, X.; Ye, Z.; Chenet, D.A.; Ye, Y.; O’Brien, K.; Hone, J.C.; Zhang, X. Edge Nonlinear Optics on a MoS Atomic Monolayer. Science 2014, 344, 488-490. [CrossRef]

20. Lu, C.-P.; Li, G.; Watanabe, K.; Taniguchi, T.; Andrei, E.Y. $\mathrm{MoS}_{2}$ : Choice Substrate for Accessing and Tuning the Electronic Properties of Graphene. Phys. Rev. Lett. 2014, 113, 156804. [CrossRef]

21. Lin, W.; Shi, Y.; Yang, X.; Li, J.; Sun, M.-T.; Xu, X.; Pullerits, T.; Liang, W.; Sun, M. Physical mechanism on exciton-plasmon coupling revealed by femtosecond pump-probe transient absorption spectroscopy. Mater. Today Phys. 2017, 3, 33-40. [CrossRef]

22. Chen, S.-L.; Liang, Y.-Z.; Hou, Y.; Wang, H.; Wu, X.; Gan, W.; Yuan, Q. Simple physics in and easy manipulating of the interfacial behavior of charged molecules on drug delivery vesicles. Mater. Today Phys. 2019, 9, 100092. [CrossRef]

23. Zhang, Y.; Lee, J.; Wang, W.; Yao, D.-X. Two-dimensional octagon-structure monolayer of nitrogen group elements and the related nano-structures. Comput. Mater. Sci. 2015, 110, 109-114. [CrossRef]

24. Kang, Y.-T.; Lu, C.; Yang, F.; Yao, D.-X. Single-orbital realization of high-temperature superconductivity in the square-octagon lattice. Phys. Rev. B 2019, 99, 184506. [CrossRef]

25. Lu, H.; Yao, Y.; Zhu, S.; Yang, Y.; Lin, L. Fabrication of free-standing octagon-shaped carbon nanofibre assembly for electrical actuation of shape memory polymer nanocomposites. Pigment. Resin Technol. 2015, 44, 157-164. [CrossRef]

26. Liu, M.; Liu, M.; She, L.; Zha, Z.; Pan, J.; Li, S.; Li, T.; He, Y.; Cai, Z.; Wang, J.; et al. Graphene-like nanoribbons periodically embedded with four- and eight-membered rings. Nat. Commun. 2017, 8, 14924. [CrossRef]

27. Yuan, J.; Zhao, M.; Yu, W.; Lu, Y.; Chen, C.; Xu, M.; Qiaoliang, B.; Loh, K.P.; Bao, Q. Raman Spectroscopy of Two-Dimensional $\mathrm{Bi}_{2} \mathrm{TexSe}_{3-x}$ Platelets Produced by Solvothermal Method. Materials 2015, 8, 5007-5017. [CrossRef]

28. Ouyang, G.; Sun, C.Q.; Zhut, W.-G. Pressure-Stiffened Raman Phonons in Group III Nitrides: A Local Bond Average Approach. J. Phys. Chem. B 2008, 112, 5027-5031. [CrossRef]

29. Bhattacharya, T.S.; Mitra, S.; Singha, S.S.; Mondal, P.K.; Singha, A. Tailoring light-matter interaction in $\mathrm{WS}_{2}$ -gold nanoparticles hybrid systems. Phys. Rev. B 2019, 100, 235438. [CrossRef]

30. Zhao, W.; Wu, Q.; Hao, H.; Wang, J.; Li, M.; Zhang, Y.; Bi, K.; Chen, Y.; Ni, Z. Plasmon-phonon coupling in monolayer $\mathrm{WS}_{2}$. Appl. Phys. Lett. 2016, 108, 131903. [CrossRef]

31. Meunier, V.; Meunier, V. First-principles Raman spectra of $\mathrm{MoS}_{2}, \mathrm{WS}_{2}$ and their heterostructures. Nanoscale 2014, 6, 5394-5401. [CrossRef]

32. Ceriotti, M.; Pietrucci, F.; Bernasconi, M. Ab initiostudy of the vibrational properties of crystalline $\mathrm{TeO}_{2}$ : The $\alpha, \beta$, and $\gamma$ phases. Phys. Rev. B 2006, 73, 104304. [CrossRef]

33. Sheng, X.-L.; Yan, Q.-B.; Ye, F.; Zheng, Q.-R.; Su, G. T-Carbon: A Novel Carbon Allotrope. Phys. Rev. Lett. 2011, 106, 155703. [CrossRef] [PubMed]

34. Zhang, J.; Wang, R.; Zhu, X.; Pan, A.; Han, C.; Li, X.; Zhao, D.; Ma, C.; Wang, W.; Su, H.; et al. Pseudo-topotactic conversion of carbon nanotubes to T-carbon nanowires under picosecond laser irradiation in methanol. Nat. Commun. 2017, 8, 1-7. [CrossRef]

35. Kresse, G.; Furthmüller, J. Efficient iterative schemes for ab initio total-energy calculations using a plane-wave basis set. Phys. Rev. B Condens. Matter. 1996, 54,11169-11186. [CrossRef] 
36. Togo, A.; Chaput, L.; Tanaka, I.; Hug, G. First-principles phonon calculations of thermal expansion in $\mathrm{Ti}_{3} \mathrm{SiC}_{2}$, $\mathrm{Ti}_{3} \mathrm{AlC}_{2}$, and $\mathrm{Ti}_{3} \mathrm{GeC}_{2}$. Phys. Rev. B 2010, 81, 174301. [CrossRef]

37. Kresse, G.; Hafner, J. Ab initiomolecular dynamics for liquid metals. Phys. Rev. B 1993, 47, 558-561. [CrossRef]

38. Kresse, G.; Hafner, J. Ab initiomolecular-dynamics simulation of the liquid-metal-amorphous-semiconductor transition in germanium. Phys. Rev. B 1994, 49, 14251-14269. [CrossRef]

39. Kresse, G.; Joubert, D. From ultrasoft pseudopotentials to the projector augmented-wave method. Phys. Rev. B 1999, 59, 1758-1775. [CrossRef]

40. Perdew, J.P.; Chevary, J.A.; Vosko, S.H.; Jackson, K.A.; Pederson, M.R.; Singh, D.J.; Fiolhais, C. Atoms, molecules, solids, and surfaces: Applications of the generalized gradient approximation for exchange and correlation. Phys. Rev. B 1992, 46, 6671-6687. [CrossRef]

41. Deng, Z.; Li, Z.; Wang, W.; She, J. Vibrational properties and Raman spectra of pristine and fluorinated blue phosphorene. Phys. Chem. Chem. Phys. 2019, 21, 1059-1066. [CrossRef] [PubMed]

42. Shegai, T.O.; Haran, G. Probing the Raman Scattering Tensors of Individual Molecules. J. Phys. Chem. B 2006, 110, 2459-2461. [CrossRef] [PubMed]

43. Kranert, C.; Sturm, C.; Schmidt-Grund, R.; Grundmann, M. Raman tensor elements of $\beta-G_{2} \mathrm{O}_{3}$. Sci. Rep . 2016, 6, 35964. [CrossRef] [PubMed]

44. Sander, T.; Eisermann, S.; Meyer, B.K.; Klar, P.J. Raman tensor elements of wurtzite ZnO. Phys. Rev. B 2012, 85, 165208. [CrossRef]

45. Zheng, W.; Zheng, R.; Huang, F.; Wu, H.; Li, F. Raman tensor of AlN bulk single crystal. Photon. Res. 2015, 3, 38-43. [CrossRef]

46. Umari, P.; Pasquarello, A.; Corso, A.D. Raman scattering intensities in $\alpha$-quartz: A first-principles investigation. Phys. Rev. B 2001, 63, 094305. [CrossRef]

47. Saboori, S.; Deng, Z.; Li, Z.; Wang, W.; She, J. $\beta$-As Monolayer: Vibrational Properties and Raman Spectra. ACS Omega 2019, 4, 10171-10175. [CrossRef]

48. Jin, M.; Zheng, W.; Ding, Y.; Zhu, Y.; Wang, W.; Huang, F. Raman Tensor of WSe 2 via Angle-Resolved Polarized Raman Spectroscopy. J. Phys. Chem. C 2019, 123, 29337-29342. [CrossRef]

49. Saboori, S.; Wang, W.; Li, Z.; She, J. Raman spectra of MXenes $Z_{2} X(X=C$ and N). Nanotechnology 2020, 31, 405708. [CrossRef]

50. Luo, G.; Wang, L.; Li, H.; Qin, R.; Zhou, J.; Li, L.; Gao, Z.; Mei, W.-N.; Lu, J.; Nagase, S. Polarized Nonresonant Raman Spectra of Graphene Nanoribbons. J. Phys. Chem. C 2011, 115, 24463-24468. [CrossRef]

51. Zhu, Y.; Zheng, W.; Wang, W.; Zhu, S.; Cheng, L.; Li, L.; Lin, Z.; Ding, Y.; Jin, M.; Huang, F. Raman tensor of layered black arsenic. J. Raman Spectrosc. 2020, 51, 1324-1330. [CrossRef]

52. Jin, M.; Zheng, W.; Ding, Y.; Zhu, Y.; Wang, W.; Huang, F. Raman Tensor of van der Waals MoSe 2 . J. Phys. Chem. Lett. 2020, 11, 4311-4316. [CrossRef] [PubMed]

53. Tuinstra, F.; Koenig, J.L. Raman Spectrum of Graphite. J. Chem. Phys. 1970, 53, 1126-1130. [CrossRef]

54. Venezuela, P.; Lazzeri, M.; Mauri, F. Theory of double-resonant Raman spectra in graphene: Intensity and line shape of defect-induced and two-phonon bands. Phys. Rev. B 2011, 84, 035433. [CrossRef]

55. Krishnan, R.S. Second-Order Raman Spectra of Crystals. Nat. Cell Biol. 1947, 160, 230-231. [CrossRef] [PubMed]

56. Wu, J.-B.; Zhang, X.; Ijäs, M.; Han, W.-P.; Qiao, X.-F.; Li, X.-L.; Jiang, D.-S.; Ferrari, A.C.; Tan, P.-H. Resonant Raman spectroscopy of twisted multilayer graphene. Nat. Commun. 2014, 5, 5309. [CrossRef] [PubMed]

Publisher's Note: MDPI stays neutral with regard to jurisdictional claims in published maps and institutional affiliations.

(C) 2020 by the authors. Licensee MDPI, Basel, Switzerland. This article is an open access article distributed under the terms and conditions of the Creative Commons Attribution (CC BY) license (http://creativecommons.org/licenses/by/4.0/). 